\title{
microRNA regulation of the expression of the estrogen receptor in endometrial cancer
}

\author{
JING ZHOU, TIANRONG SONG，SHIPENG GONG，MEI ZHONG and GUIDONG SU \\ Department of Obstetrics and Gynecology, Nanfang Hospital, Southern Medical University, \\ Guangzhou 510515, Guangdong Province, P.R. China
}

Received November 30, 2009; Accepted February 9, 2010

DOI: $10.3892 / \mathrm{mmr} 00000269$

\begin{abstract}
RNAs (miRNAs) are non-coding RNA transcripts thought to be instrumental in controlling eukaryotic cell function. This study aimed to identify miRNAs associated with the differential expression of estrogen receptor $\alpha$ $(\mathrm{ER} \alpha)$ and progesterone receptor $(\mathrm{PR})$ in uterine endometrial cancer. First, the pathology of Ishikawa and KLE cell lines was identified by transplant and biopsy. Expression levels of $\mathrm{ER} \alpha$ and $\mathrm{PR}$ and their response to estrogen and progesterone were examined, and total RNA was isolated to identify differentially expressed miRNAs by microarray analysis. miRNAs targeting ESR1 and PGR were predicted by miRANDA and TargetScan software, and their expression levels in cell lines and in patient samples were validated by real-time RT-PCR. Ishikawa and KLE represent estrogen-dependent and estrogen-independent endometrial cancers, respectively. As identified by miRNA microarray, 126 miRNAs were differentially expressed between the two cell lines; hsa-miR-100 and hsa-miR-99a were predicted to target ESR1, and hsa-miR-378 and hsa-miR-768-3p to target PGR. The differential expression of these miRNAs between Ishikawa and KLE was consistent in vivo and in vitro. Hsa-miR-100 was significantly down-regulated in the estrogen-dependent endometrial cancer samples as compared to the estrogen-independent samples and thus has the potential to target ESR1.
\end{abstract}

\section{Introduction}

Endometrial cancer (EC) is one of the three most common types of cancer of the female genital tract. In 1983, it was divided into two categories based on clinicopathological features (1). It was discovered that one of the categories was more often related to unopposed estrogen stimulation than

Correspondence to: Dr Guidong Su, Department of Obstetrics and Gynecology, Nanfang Hospital, Southern Medical University, Guangzhou 510515, Guangdong Province, P.R. China

E-mail: suguidong@gmail.com

Key words: endometrial cancer, estrogen dependency, estrogen receptor $\alpha$, microRNA, target gene, microarray, real-time RT-PCR the other. They were therefore termed estrogen-dependent EC (EDEC) and estrogen-independent EC (EIEC). Although EIEC accounts for only $20-30 \%$ of the total cases, it displays lower differentiation, deeper myometrial invasion, higher incidence of lymph node metastasis and a higher recurrence rate, and therefore has a worse prognosis (2). In most cases, the expression of ER $\alpha$ and PR differs significantly between EIEC and EDEC, and is considered an important predictor of prognosis (3).

microRNAs (miRNAs) are 19-25 nt non-coding RNA transcripts thought to be instrumental in controlling eukaryotic cell function via post-transcriptional repression of multiple target mRNA genes (4). Different tissues or cells express different miRNAs in different phases. One miRNA modulates more than one hundred target genes, including transcription factors, cytokines and receptors. miRNAs and their target genes compose a complicated modulation network. Recent studies have found that miRNAs are associated with carcinogenesis and tumor progression through their effects on the generation, apoptosis, invasion, angiogenesis and metastasis of tumor cells (5).

microRNAs have rarely been reported in endometrial cancer research. Information with regards to the difference in miRNA expression between EDEC and EIEC and their association with carcinogenesis remains elusive. In this study, miRNAs that were differentially expressed between the two types of endometrial cancers were screened using microarray analysis. Potential miRNA candidates associated with ER $\alpha$ and PR expression were predicted using prediction software, and were validated in patient samples.

\section{Materials and methods}

Cell lines. The human endometrial cancer cell lines KLE and Ishikawa were a kind gift from Professor Y.J. Feng of the Fudan University Obstetrics and Gynecology Hospital, and Professor J.L. Wang of the Obstetrics and Gynecology Department of Peking University Second Hospital.

Fresh-frozen samples. Samples were available from 153 endometrial cancer patients who underwent surgery at Nanfang Hospital between 2006 and 2008. Based on medical history and pathological reports, 24 patients were selected and categorized in the EIEC group, and 24 age-matched 
patients were selected for the EDEC group. Each patient in the EDEC group had a history of one or more high estrogen exposure factors: later menopause ( $\geq 55$ years of age), obesity (BMI $\geq 24$ ), diabetes mellitus, hormone replacement or infertility. Patients in the EIEC group had no exposure factors. Patients in the EDEC group had a mean age of 60.4 years, and pathological reports showed that all samples in this group were of well-differentiated endometrioid cancer and were ER $\alpha$ - and PR-positive; 15 patients were in stage Ic and 9 were in stage Ib. Patients in the EIEC group had a mean age of 61 years, and the samples were ER $\alpha$ - and PR-negative endometrial cancer, including 7 moderateto-poorly differentiated and 8 poorly differentiated serous cancers, 3 poorly differentiated endometrioid cancers with serous-like change, 6 poorly differentiated endometrioid cancers, 6 in stage Ib, 10 in stage Ic, 3 in stage II and 5 in stage III.

Cell culture. KLE and Ishikawa cell lines were cultured in Phenol red-free (phenol red has slight estrogen-like activity) Dulbecco's modified Eagle's medium (Gibco, USA) supplemented with dextran-treated (dextran absorbs estrogen and progesterone from the serum, contributing to the estrogen progesterone-free culture condition) $10 \%$ fetal calf serum (Hycolon, USA) and 1X antibiotic/antifungal solution (Sigma, USA). Medium was replaced every 2-3 days. Cells were digested with $0.25 \%$ trypsinase $+0.004 \%$ EDTA (Gibco) and expanded at a ratio of 1:3. Cells used for the following steps were collected in the mid-logarithmic growth phase.

Pathological identification of tumors derived from the cell lines. BALB/C nude mice (Experimental Animal Centre of Peking University, China) were subcutaneously injected into the right armpit with $10^{7} \mathrm{KLE}$ or Ishikawa cells. Mice were sacrificed, and tumors were harvested upon reaching $0.5 \mathrm{~cm}$ in diameter. Tumors were fixed with $4 \%$ formalin, embedded with paraffin, sectioned into $4-\mu \mathrm{m}$ slices and stained with H\&E. Slides were examined by an experienced pathologist to identify the pathological type and differentiation grade.

Identification of the expression of ER $\alpha$ and PR using immunochemical staining. Cells were collected in the mid-logarithmic growth phase, fixed with $4 \%$ formalin, embedded with paraffin, cut into $4-\mu \mathrm{m}$ sections and stained using a streptavidin-peroxidase (SP) one-step staining method including primary antibody p63 mouse anti-human IgG monoclonal antibody and the SP immunochemistry kit (Beijing Zhongshan Goldenbridge Biotechnology Co., Ltd., China). Both cell lines were probed with antibodies to ER $\alpha$ and PR.

Identification of the response to estrogen and progesterone using the CCK-8 method. The effects of $17 \beta$-estrogen (17 $\beta$-E2) (Sigma) at concentrations of $0,0.0001,0.001$, $0.01,0.1$ and $1.0 \mu \mathrm{mol} / 1$ and medroxyprogesterone acetate (MPA) (Sigma) at concentrations of $0,0.001,0.01,0.1$, 1.0 and $10 \mu \mathrm{mol} / 1$ on the growth of the two cell lines were examined using the Cell Counting kit-8 (Dojindo Molecular
Technologies, Inc., Japan) following the manufacturer's instructions. The reaction time was $72 \mathrm{~h}$. OD values were determined at a 450-nm wavelength using ELISA (Varioskan Flash; Thermo Scientific, USA). The OD value was proportional to the cell density.

microRNA microarray assay. Total RNA was isolated using TRIzol (Invitrogen, USA) following the manufacturer's instructions. An RNA quality test and the microarray assay were performed using a service provider (LC Sciences, USA). For each sample, 2-5 $\mu \mathrm{g}$ of total RNA was used, which was size fractionated using a YM-100 Microcon centrifugal filter (Millipore, USA). The small RNAs (<300 nt) isolated were 3 -extended with a poly(A) tail using poly(A) polymerase. An oligonucleotide tag was then ligated to the poly(A) tail for later fluorescent dye staining. Two different tags were used for the two RNA samples in dual-sample experiments. Hybridization was performed overnight on a $\mu$ Paraflo $^{\mathrm{TM}}$ microfluidic chip (LC Sciences) using a micro-circulation pump (Atactic Technologies, USA). Each detection probe on the microfluidic chip consisted of a chemically modified nucleotide coding segment complementary to the target miRNA (from miRBase, version 11.0 microRNA, Sanger, http://microrna.sanger.ac.uk/sequences/) and a spacer segment of polyethylene glycol to extend the coding segment away from the substrate. The detection probes were made by in situ synthesis using photo-generated reagent (PGR) chemistry. The hybridization melting temperatures were balanced by chemical modifications of the detection probes. Hybridization was performed using $100 \mu \mathrm{l} 6 \mathrm{X} \mathrm{SSPE}$ buffer $(0.90 \mathrm{M} \mathrm{NaCl}$, $60 \mathrm{mM} \mathrm{Na} \mathrm{HPO}_{4}, 6 \mathrm{mM}$ EDTA, $\mathrm{pH}$ 6.8) containing 25\% formamide at $34^{\circ} \mathrm{C}$. Products were labeled with fluorescence using tag-specific $\mathrm{Cy} 3$ and $\mathrm{Cy} 5$ dyes. Hybridization images were collected using a laser scanner (GenePix 4000B; Molecular Devices, USA) and digitized using Array-Pro image analysis software (Media Cybernetics, USA). The data were analyzed by first subtracting the background and then normalizing the signals using a locally weighted regression filter (LOWESS). For two-color experiments, the ratio of the two sets of detected signals ( $\log 2$ transformed, balanced) and p-values of the t-test were calculated. Statistical significance was defined by $\mathrm{p}$-values $<0.01$.

Prediction of microRNAs targeting ESR1 or PGR in endometrial cancer. miRNAs targeting ESR1 or PGR were predicted using miRanda and TargetScan prediction programs and were compared with the microarray results. It was expected that the expression of miRNAs would be inversely related to their target mRNAs.

Validation of the expression of microRNAs by RT-PCR. The remaining Ishikawa and KLE cell line samples (after pathological identification) and the 48 endometrial cancer fresh-frozen samples were cut into $20-\mu \mathrm{m}$ sections, H\&E stained and placed in DEPC water to avoid drying. Cancer tissues were dissected from the sections with a fine needle tip under a x10 visual field of an inverted microscope (Olympus, Japan). The cancer tissues were transferred to an RNase-free EP tube. All procedures were performed in an RNase-free environment. 
Total RNA from the samples was isolated with TRIzol (Invitrogen) following the manufacturer's instructions. The miRNAs selected for validation were hsa-miR-100, hsamiR-99a, hsa-miR-378 and hsa-miR-768-3P. hsa-let-7-b was used as an internal control. The primers for RT-PCR are listed in Table I. After reverse transcription, cDNAs were amplified with miRNA-specific forward and reverse primers and SYBR GreenER ${ }^{\mathrm{TM}}$ qPCR reagents (Invitrogen) in an ABI PRISM 7500 sequence detection system (Applied Biosystems, USA). Expression of the tested miRNAs was calculated based on the ratio of sample $\mathrm{Ct}$ values to internal control values.

Statistical analysis. Data were analyzed using SPSS software version 15.0. A two-independent-sample t-test was performed, and a p-value $<0.05$ was considered statistically significant.

\section{Results}

Pathological determination of the tumors derived from the cell lines. Ishikawa cell tumors presented characteristics of well-differentiated endometrioid cancer, including aligned glandular structures with little interstitial substance, and cubic or column-shaped cancer cells with a round or oval nucleus and clear nucleoli. KLE cell tumors presented characteristics of poorly differentiated endometrioid cancer with no glandular structure and disorderly arranged cancer cells with heteromorphic nuclei and karyodieresis (Fig. 1).

Expression of ER $\alpha$ and $P R$. Ishikawa cells presented high expression of $\mathrm{ER} \alpha$ and PR (positive rates of 95 and $90 \%$, respectively), while KLE cells presented low expression of $\mathrm{ER} \alpha$ and PR (positive rates of $<1 \%$ ) (Fig. 2).

Response of cells to estrogen and progesterone. The density of the Ishikawa cells increased in relation to the concentration of $17 \beta-\mathrm{E} 2$ and peaked at $0.1 \mu \mathrm{mol} / 1$. The $17 \beta-\mathrm{E} 2$ had no significant effect on KLE cells. Higher doses of MPA treatment decreased the density of the Ishikawa cells, but had no effect on KLE cells.

Results of microRNA microarray assay. Expression of 59 miRNAs was higher, and expression of 67 miRNAs was lower in the KLE cell line when compared to the Ishikawa cell line (partial list provided in Table II).

Results of microRNA-target gene prediction. In both cell lines, hsa-miR-100 and hsa-miR-99a were predicted to target ESR1, and hsa-miR-378 and hsa-miR-768-3P were predicted to target PGR.

Validation by RT-PCR assay. Expression of the four miRNAs was significantly different in the two cell lines both in vitro and in vivo $(\mathrm{p}<0.01)$, but only hsa-miR-100 was differentially expressed between the two groups of samples representing EDEC and EIEC ( $\mathrm{p}=0.002)$. Expression of the other three miRNAs exhibited no statistically significant difference between the two groups of samples $(\mathrm{p}>0.05)$ (Table III). 


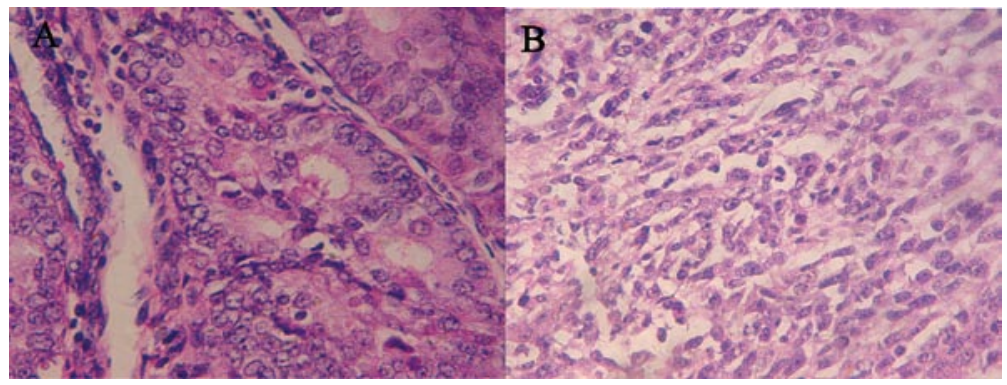

Figure 1. Tumor sections (H\&E stained, $\mathrm{x} 40$ visual field). (A) Ishikawa presented characteristics of well-differentiated endometrioid cancer, including aligned glandular structure with little interstitial substance, cubic or column-shaped cancer cells with round or oval nuclei and clear nucleoli. (B) KLE presented characteristics of poorly differentiated endometrioid cancer with no glandular structure, disorderly arranged cancer cells with heteromorphic nuclei and karyodieresis.

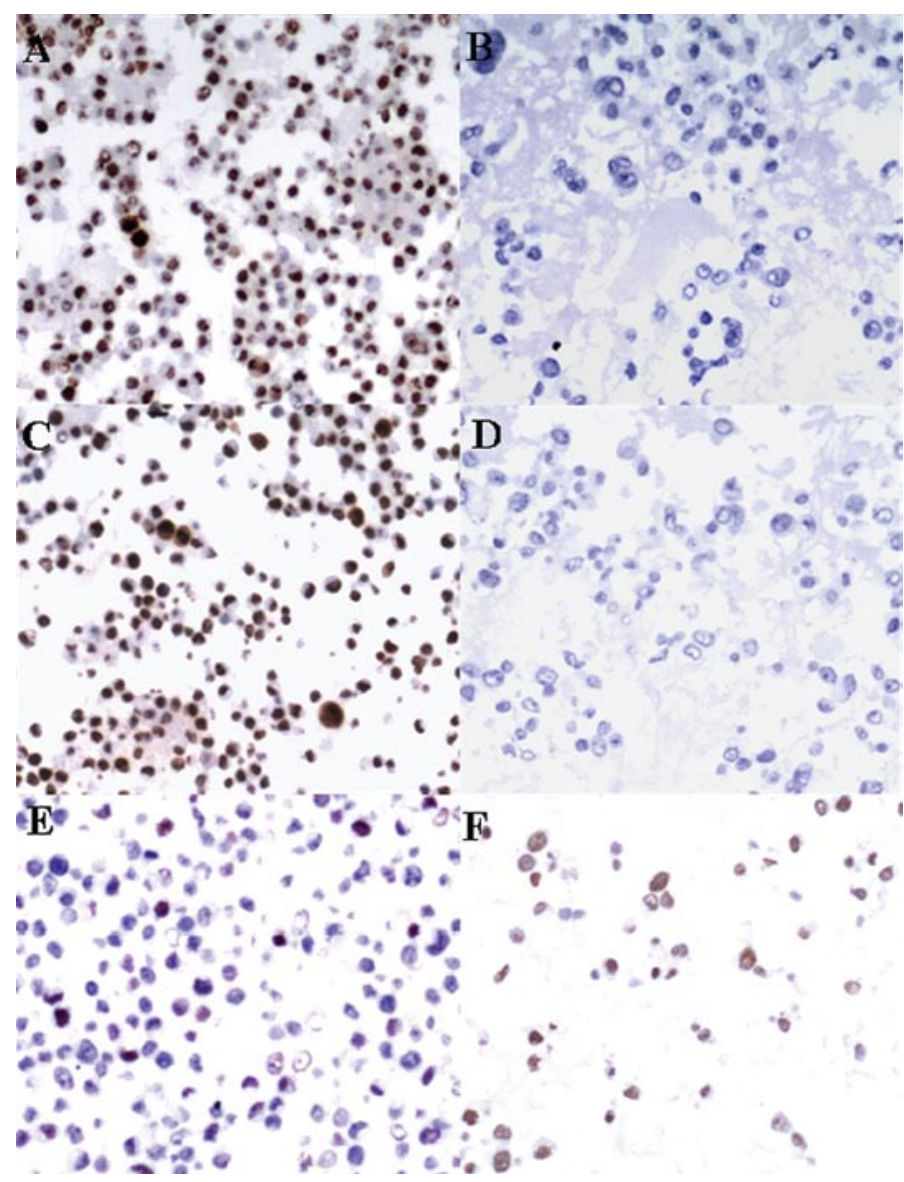

Figure 2. Immunochemical staining for $\mathrm{ER} \alpha, \mathrm{PR}$ and $\mathrm{p} 53$ with a positive rate. All three antigens were expressed in the nuclei. Brown color represents positive staining, while blue represents negative. Ishikawa presented high expression of ER $\alpha(95 \%$; A) and PR (90\%; C) and low expression of p53 (35\%; E), while KLE presented low expression of $\mathrm{ER} \alpha(<1 \%$; B) and PR (<1\%; D) and high expression of p53 (90\%; F).

A

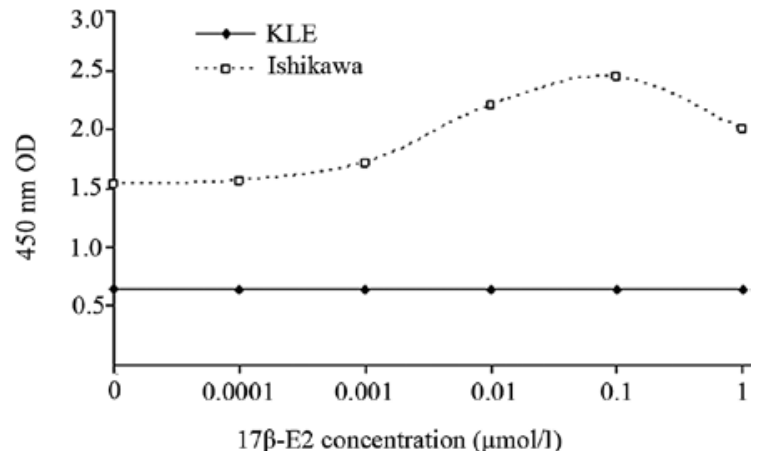

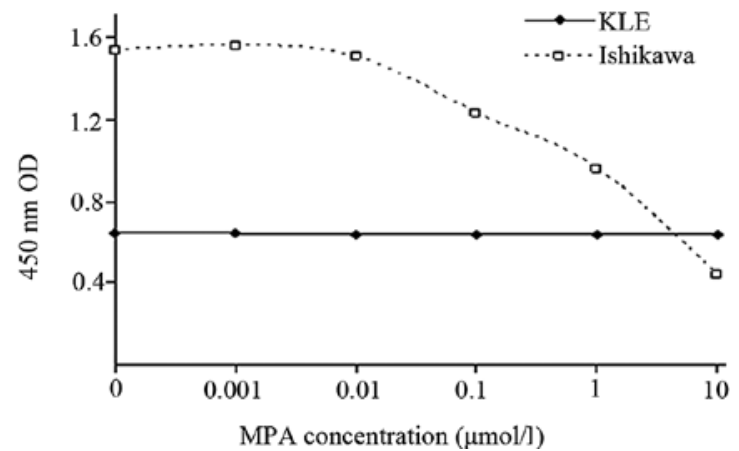

Figure 3. Concentration-OD curves of two cell lines for different concentrations. (A) Concentration-OD curves of two cell lines with $17 \beta$-E2 for $72 \mathrm{~h}$. (B) Concentration-OD curves of two cell lines with MPA for $72 \mathrm{~h}$. 
Table II. microRNAs exhibiting significant differential expression between Ishikawa and KLE cells.

\begin{tabular}{clrrr}
\hline No. & Probe_ID & KLE signal & Ishikawa signal & $\log _{2}($ KLE/Ishikawa $)$ \\
\hline 1 & hsa-miR-200b & 40.79 & $23,444.08$ & 9.12 \\
2 & hsa-miR-200c & 49.74 & $25,417.78$ & 9.08 \\
3 & hsa-miR-146a & $2,030.28$ & 5.04 & -8.64 \\
4 & hsa-miR-429 & 11.68 & $1,943.77$ & 7.39 \\
5 & hsa-miR-145 & $1,348.11$ & 9.58 & -7.12 \\
6 & hsa-miR-200a & 9.74 & $1,083.57$ & 6.78 \\
7 & hsa-miR-143 & 931.85 & 9.44 & -6.55 \\
8 & hsa-miR-146b-5p & $1,059.26$ & 15.85 & -6.13 \\
9 & hsa-miR-335 & 8.51 & 412.77 & 5.79 \\
10 & hsa-miR-744 & 5.00 & 251.09 & 5.75 \\
43 & hsa-miR-378 & 266.90 & 55.62 & -2.31 \\
52 & hsa-miR-100 & $1,385.59$ & 348.38 & -2.01 \\
59 & hsa-miR-768-3p & $1,476.21$ & 413.78 & -1.90 \\
76 & hsa-miR-99a & $2,817.43$ & $1,001.68$ & -1.43 \\
\hline
\end{tabular}

Table III. Results of real-time RT-PCR conducted in cell lines both in vitro (group 1) and in vivo (group 2) and samples from patients (group 3 ).

\begin{tabular}{|c|c|c|c|c|}
\hline & $\mathrm{Ct}_{100} / \mathrm{Ct}_{\text {let7b }}$ & $\mathrm{Ct}_{99 \mathrm{a}} / \mathrm{Ct}_{\mathrm{let} 7 \mathrm{~b}}$ & $\mathrm{Ct}_{378} / \mathrm{Ct}_{\text {let7b }}$ & $\mathrm{Ct}_{768-3 \mathrm{p}} / \mathrm{Ct}_{\text {let7b }}$ \\
\hline \multicolumn{5}{|l|}{ Group 1} \\
\hline Ishikawa (in vitro) & $1.091 \pm 0.004$ & $1.071 \pm 0.008$ & $1.085 \pm 0.005$ & $1.036 \pm 0.005$ \\
\hline KLE (in vitro) & $1.020 \pm 0.007$ & $1.028 \pm 0.010$ & $1.018 \pm 0.011$ & $0.948 \pm 0.008$ \\
\hline p-value & $<0.001$ & $<0.001$ & $<0.001$ & $<0.001$ \\
\hline \multicolumn{5}{|l|}{ Group 2} \\
\hline Ishikawa (in vivo) & $1.113 \pm 0.008$ & $1.050 \pm 0.011$ & $1.057 \pm 0.007$ & $1.025 \pm 0.009$ \\
\hline KLE (in vivo) & $1.054 \pm 0.005$ & $1.022 \pm 0.007$ & $1.010 \pm 0.005$ & $0.989 \pm 0.004$ \\
\hline p-value & $<0.001$ & 0.002 & $<0.001$ & $<0.001$ \\
\hline \multicolumn{5}{|l|}{ Group 3} \\
\hline Type I (n=12) & $1.029 \pm 0.009$ & $0.578 \pm 0.152$ & $0.855 \pm 0.085$ & $0.926 \pm 0.034$ \\
\hline Type II (n=12) & $1.007 \pm 0.007$ & $0.611 \pm 0.088$ & $0.752 \pm 0.078$ & $0.970 \pm 0.048$ \\
\hline p-value & 0.002 & 0.120 & 0.096 & 0.152 \\
\hline
\end{tabular}

Table IV. Several of the differentially expressed microRNAs between Ishikawa and KLE cells and their association to tumors.

\begin{tabular}{|c|c|c|}
\hline ID & Signal difference ( $\log _{2}$ KLE/Ishikawa) & Association to tumors (reference) \\
\hline hsa-miR-34a & -2.30 & Potential anti-oncogene, regulated by p53 $(9,10)$ \\
\hline hsa-miR-16 & -0.46 & $\begin{array}{l}\text { Target BCL2, able to induce apoptosis, could be used in the } \\
\text { therapy of tumors which overexpress Bcl-2 (11) }\end{array}$ \\
\hline hsa-miR-21 & 0.62 & Oncogene (12) \\
\hline hsa-miR-126 & 5.38 & $\begin{array}{l}\text { Target VCAM-1, VCAM-1 overexpressed in tumor was } \\
\text { associated with T lymphocyte-mediated tumor immunoescape } \\
\text { (13) }\end{array}$ \\
\hline hsa-miR-378 & 2.31 & $\begin{array}{l}\text { Target Sufu and Fus-1, able to promote development, } \\
\text { survival and angiogenesis of tumor (14) }\end{array}$ \\
\hline hsa-miR-222 & 1.70 & $\begin{array}{l}\text { Overexpressed in thyroid papillary carcinoma, } \\
\text { regulates the level of protein p27Kip1 and cell cycle (15) }\end{array}$ \\
\hline hsa-miR-221 & 1.40 & $\begin{array}{l}\text { Overexpressed in thyroid papillary carcinoma, } \\
\text { regulates the level of protein p27Kip1 and cell cycle (15) }\end{array}$ \\
\hline
\end{tabular}




\section{Discussion}

The parameters for distinguishing the different types of endometrial cancer have thus far been poorly defined. Bokhman (1) related EDEC to high exposure to unopposed estrogen. In other words, EDEC may be promoted by estrogen and inhibited by progesterone, because progesterone antagonizes estrogen and promotes differentiation (6). The carcinogenesis of EIEC has no correlation with estrogen, which is consistent with our findings. This explains why progesterone, although it is an effective agent for treating EDEC, has no effect on EIEC (6,7). According to long-term clinicopathological observations, most EDEC samples are well-differentiated and express ER $\alpha$ and PR in contrast to EIEC samples $(6,8)$. We therefore concluded that Ishikawa and KLE cells represent EDEC and EIEC, respectively.

Huge differences in miRNA profile were revealed by miRNA microarray analysis of Ishikawa and KLE cell lines, which represent EDEC and EIEC, respectively. Some miRNAs have been reported to be associated with tumorigenesis (Table IV). To better understand the importance of characteristic miRNA profiles in different endometrial cancer phenotypes, it is of utmost importance to identify target genes.

The RT-PCR assay conducted on Ishikawa and KLE cells cultured in vitro confirmed that the differential expression of hsa-miR-100, hsa-miR-99a, hsa-miR-378 and hsa-miR-768-3p was consistent with the microarray assay. The results from the RT-PCR assay of the two cell lines, both cultured and grown in nude mice, demonstrated that the differential expression of the four miRNAs was consistent in vitro and in vivo.

The real-time RT-PCR analysis of fresh-frozen samples from patients demonstrated that expression of hsa-miR-100 was significantly lower in the EDEC than in the EIEC group. However, expression of hsa-miR-99a, hsa-miR-378 and hsamiR-768-3p exhibited no statistically significant difference. It has been reported that ER $\alpha$ is highly expressed in EDEC compared to EIEC. Hsa-miR-100 was predicted to target ESR1 by prediction software, which means they follow the rules of miRNA-target interaction based on homology and structural criteria $(9,16)$. This prediction led us to hypothesize that hsa-miR-100 may target ESR1, indicating that hsa-miR-100 regulates the expression of ER $\alpha$. Considering the important role of ER $\alpha$ in carcinogenesis and the biological behavior of endometrial cancer (17), hsa-miR-100 may be a promising candidate for further investigation and may contribute to the pathological diagnosis, treatment and prognosis of endometrial carcinoma.

\section{Acknowledgements}

We would like to thank LC Sciences (USA) for providing the microRNA microarray assay service.

\section{References}

1. Bokhman JV: Two pathogenetic types of endometrial carcinoma. Gynecol Oncol 15: 10-71, 1983.

2. Gründker C, Günthert AR and Emons G: Hormonal heterogeneity of endometrial cancer. Adv Exp Med Biol 630: 166-188, 2008.

3. Uharcek P: Prognostic factors in endometrial carcinoma. J Obstet Gynaecol Res 34: 776-783, 2008.

4. Filip A: miRNA - new mechanisms of gene expression control. Postepy Biochem 53: 413-419, 2007.

5. Bueno MJ, de Castro IP and Malumbres M: Control of cell proliferation pathways by microRNAs. Cell Cycle 7: 3143-3148, 2008.

6. Fujimoto J, Hirose R, Sakaguchi H and Tamaya T: Estrogen dependency in uterine endometrial cancers. Oncology 55 (Suppl 1): 53-59, 1998.

7. Matsumoto K and Noguchi S: Hormone therapy - recent advances. Gan To Kagaku Ryoho 14: 2816-2823, 1987.

8. Vihko R, Alanko A, Isomaa V and Kauppila A: The predictive value of steroid hormone receptor analysis in breast, endometrial and ovarian cancer. Med Oncol Tumor Pharmacother 3: 197-210, 1986.

9. Bentwich I: Prediction and validation of microRNAs and their targets. FEBS Lett 579: 5904-5910, 2005.

10. Zhao Y, Ransom JF, Li A, et al: Dysregulation of cardiogenesis, cardiac conduction, and cell cycle in mice lacking miRNA-1-2. Cell 129: 1-15, 2007.

11. Kumamoto K, Spillare EA, Fujita K, et al: Nutlin-3a activates p53 to both down-regulate inhibitor of growth 2 and up-regulate mir-34a, mir-34b and mir-34c expression, and induce senescence. Cancer Res 68: 3193-3203, 2008.

12. Tazawa H, Tsuchiya N, Izumiya M, et al: Tumor-suppressive miR-34a induces senescence-like growth arrest through modulation of the E2F pathway in human colon cancer cells. Proc Natl Acad Sci USA 104: 15472-15477, 2007.

13. Xia L, Zhang D, Du R, et al: miR-15b and miR-16 modulate multidrug resistance by targeting BCL2 in human gastric cancer cells. Int J Cancer 123: 372-379, 2008.

14. Asangani IA, Rasheed SA, Nikolova DA, et al: microRNA-21 (miR-21) post-transcriptionally down-regulates tumor suppressor Pdcd4 and stimulates invasion, intravasation and metastasis in colorectal cancer. Oncogene 27: 2128-2136, 2008.

15. Harris TA, Yamakuchi M, Ferlito M, et al: microRNA-126 regulates endothelial expression of vascular cell adhesion molecule 1. Proc Natl Acad Sci USA 105: 1516-1521, 2008.

16. John B, Sander C and Marks DS: Prediction of human microRNA targets. Methods Mol Biol 342: 101-113, 2006.

17. Tan DS, Lambros MB, Marchiò $C$ and Reis-Filho JS: ESR1 amplification in endometrial carcinomas: hope or hyperbole? J Pathol 216: 271-274, 2008. 